\title{
Some computational formulas related the Riemann zeta-function tails
}

Hongmin $\mathrm{Xu}^{*}$

\author{
"Correspondence: \\ xuhongmin@bipt.edu.cn \\ Department of Mathematics and \\ Physics, Beijing Institute of \\ Petrochemical Technology, Beijing, \\ 102617, P.R. China
}

\begin{abstract}
In this paper we present two computational formulae for one kind of reciprocal sums related to the Riemann zeta-function at integer points $s=4,5$, which answers an open problem proposed by Lin (J. Inequal. Appl. 2016:32, 2016).
\end{abstract}

MSC: $11 \mathrm{~B} 83 ; 11 \mathrm{MO6} ; 11 \mathrm{~J} 70$

Keywords: Riemann zeta-function; inequality; continued fraction; telescoping method; multiple-correction method

\section{Introduction and main results}

Let $\left(a_{k}\right)_{k \geq 1}$ be a strictly increasing positive sequence such that

$$
\sum_{k=1}^{\infty} \frac{1}{a_{k}}<+\infty
$$

Many authors study the computational formula for infinite sums of reciprocal $a_{k}$,

$$
\left\lfloor\left(\sum_{k=n}^{\infty} \frac{1}{a_{k}}\right)^{-1}\right\rfloor, \quad n \in \mathbb{N},
$$

where $\lfloor x\rfloor$ denotes the integer part of $x$.

For example, let $\left(F_{k}\right)$ be the famous Fibonacci sequence: $F_{k+1}=F_{k}+F_{k-1}$ with the initial values $F_{0}=0$ and $F_{1}=1$. Ohtsuka and Nakamura [2] showed that

$$
\begin{aligned}
& \left\lfloor\left(\sum_{k=n}^{\infty} \frac{1}{F_{k}}\right)^{-1}\right\rfloor= \begin{cases}F_{n-2} & \text { if } n \geq 2 \text { is even, } \\
F_{n-2}-1 & \text { if } n \geq 1 \text { is odd, }\end{cases} \\
& \left\lfloor\left(\sum_{k=n}^{\infty} \frac{1}{F_{k}^{2}}\right)^{-1}\right\rfloor= \begin{cases}F_{n-1} F_{n}-1 & \text { if } n \geq 2 \text { is even, } \\
F_{n} F_{n-1} & \text { if } n \geq 1 \text { is odd. }\end{cases}
\end{aligned}
$$

$\mathrm{Xu}$ and Wang [3] obtained a complex computational formula for $a_{k}=F_{k}^{3}$.

Zhang and Wang [4] studied this problem for the Pell numbers $P_{k}$ and showed that

$$
\left\lfloor\left(\sum_{k=n}^{\infty} \frac{1}{P_{k}}\right)^{-1}\right\rfloor= \begin{cases}P_{n-1}+P_{n-2} & \text { if } n \geq 2 \text { is even, } \\ P_{n-1}+P_{n-2}-1 & \text { if } n \geq 1 \text { is odd, }\end{cases}
$$

(c) $2016 \mathrm{Xu}$. This article is distributed under the terms of the Creative Commons Attribution 4.0 International License (http://creativecommons.org/licenses/by/4.0/), which permits unrestricted use, distribution, and reproduction in any medium, provided you give appropriate credit to the original author(s) and the source, provide a link to the Creative Commons license, and indicate if changes were made. 
where the Pell numbers $P_{k}$ are defined by $P_{0}=0, P_{1}=1$, and the recurrence relation $P_{k+1}=$ $2 P_{k}+P_{k-1}$.

For some other results related to recursive sequences, recursive polynomials, and their promotion forms, see [5-13] and references therein.

Very recently, Lin [1] investigated the related problem for the sequence $a_{k}=k^{s}$ with integer $s \geq 2$ and showed the following two interesting identities:

$$
\begin{aligned}
& \left\lfloor\left(\sum_{k=n}^{\infty} \frac{1}{k^{2}}\right)^{-1}\right\rfloor=n-1, \\
& \left\lfloor\left(\sum_{k=n}^{\infty} \frac{1}{k^{3}}\right)^{-1}\right\rfloor=2 n(n-1) .
\end{aligned}
$$

This is an important problem, which has a close relationship with the Riemann zetafunction $\zeta(s)$. Lin noted that there does not exist an integer-coefficient polynomial $q(x)$ of degree 3 such that the following identity holds:

$$
\left\lfloor\left(\sum_{k=n}^{\infty} \frac{1}{k^{4}}\right)^{-1}\right\rfloor=q(n)
$$

In [1], Lin declared that giving a precise calculation formula for $\left(\sum_{k=n}^{\infty} \frac{1}{k^{s}}\right)^{-1}$ with $s=4$ is a very complicated problem. In this paper, we tackle this open problem.

Theorem 1 For all integer $n \geq 2$, we have the identity

$$
\left\lfloor\left(\sum_{k=n}^{\infty} \frac{1}{k^{4}}\right)^{-1}\right\rfloor=-1+4 n-5 n^{2}+3 n^{3}+\left\lfloor\frac{(2 n+1)(n-1)}{4}\right\rfloor .
$$

Furthermore, for $a_{k}=k^{5}$, we also have an analogous computational formula.

Theorem 2 For all integer $n \geq 4$, we have

$$
\left\lfloor\left(\sum_{k=n}^{\infty} \frac{1}{k^{5}}\right)^{-1}\right\rfloor=-5 n+9 n^{2}-8 n^{3}+4 n^{4}+\left\lfloor\frac{(n+1)(n-2)}{3}\right\rfloor .
$$

\section{Proof of Theorem 1}

Assume that

$$
g(n)-g(n+1)<\frac{1}{n^{4}}<f(n)-f(n+1),
$$

and $f(\infty)=g(\infty)=0$. Summing the inequalities from $n$ to $\infty$, we have

$$
g(n)<\sum_{k=n}^{\infty} \frac{1}{k^{4}}<f(n) .
$$

These inequalities allow us to study the computational formulas of Theorem 1 . The problem of finding the functions $f(n), g(n)$ (or $F(n), G(n)$ in Section 3 ) is transformed into 
solving the finite continued fraction approximation solution of difference equation for 'large' n:

$$
y(n)-y(n+1)=\frac{1}{n^{4}} .
$$

We will apply the multiple-correction method (see [14-16]) and solve it as follows.

Step 1 (The initial correction) Choosing $\eta_{0}(n)=\frac{b}{n^{3}+a_{2} n^{2}+a_{1} n+a_{0}}$ and developing the expression $\eta_{0}(n)-\eta_{0}(n+1)-\frac{1}{n^{4}}$ into power series expansion in $1 / n$, we easily obtain

$$
\begin{aligned}
\eta_{0}(n) & -\eta_{0}(n+1)-\frac{1}{n^{4}} \\
= & (3 b-1) \frac{1}{n^{4}}+\left(-6 b-4 a_{2} b\right) \frac{1}{n^{5}}+\left(10 b-5 a_{1} b+10 a_{2} b+5 a_{2}^{2} b\right) \frac{1}{n^{6}} \\
& +\left(-15 b-6 a_{0} b+15 a_{1} b-20 a_{2} b+12 a_{1} a_{2} b-15 a_{2}^{2} b-6 a_{2}^{3} b\right) \frac{1}{n^{7}}+O\left(\frac{1}{n^{8}}\right) .
\end{aligned}
$$

If $b=\frac{1}{3}, a_{2}=-\frac{3}{2}, a_{1}=\frac{5}{4}, a_{0}=-\frac{3}{8}$, then we can get the approximation solution

$$
g(n)=\frac{b}{n^{3}+a_{2} n^{2}+a_{1} n+a_{0}}
$$

of difference equation (2.2), which is the best possible rational approximation solution of such structure as $n$ tends to infinity.

Step 2 (The first correction) Choose $\eta_{1}(n)=\frac{b}{n^{3}+a_{2} n^{2}+a_{1} n+a_{0}+\frac{u}{x+\nu}}$ and developing the expression $\eta_{1}(n)-\eta_{1}(n+1)-\frac{1}{n^{4}}$ into power series expansion in $1 / n$, we easily obtain

$$
\eta_{1}(n)-\eta_{1}(n+1)-\frac{1}{n^{4}}=\left(-\frac{7}{16}-\frac{7}{3} u\right) \frac{1}{n^{8}}+\frac{4}{3}(u+2 u v) \frac{1}{n^{9}}+O\left(\frac{1}{n^{10}}\right) .
$$

If $u=-\frac{3}{16}, v=-\frac{1}{2}$, then we can get the approximation solution

$$
f(n)=\frac{b}{n^{3}+a_{2} n^{2}+a_{1} n+a_{0}+\frac{u}{x+\nu}}
$$

of difference equation (2.2), which has a better approximation rate than $g(n)$ for 'large' $n$.

So we can get following inequalities necessary in the proofs of our theorems.

Lemma 1 Let

$$
f(n)=\frac{\frac{8}{3}}{-1+10 n-12 n^{2}+8 n^{3}-\frac{3}{2 n-1}} .
$$

Then, for $n \geq 2$,

$$
f(n)-f(n+1)>\frac{1}{n^{4}}
$$


Proof We easily check that

$$
f(n)-f(n+1)-\frac{1}{n^{4}}=\frac{1}{n^{4}(1+n)\left(1+n+n^{2}\right)\left(-1+2 n-2 n^{2}+n^{3}\right)} .
$$

Note that $-1+2 n-2 n^{2}+n^{3}=(-2+n)\left(2+n^{2}\right)+3$, so the above polynomial is positive for $n \geq 2$. Then, for $n \in \mathbb{N}$,

$$
f(n)-f(n+1)-\frac{1}{n^{4}}>0 .
$$

Lemma 2 Let

$$
g(n)=\frac{8}{3\left(-3+10 n-12 n^{2}+8 n^{3}\right)} .
$$

Then, for $n \in \mathbb{N}$,

$$
g(n)-g(n+1)<\frac{1}{n^{4}}
$$

Proof We have

$$
g(n)-g(n+1)-\frac{1}{n^{4}}=\frac{-28 n^{2}+9}{n^{4}\left(-3+10 n-12 n^{2}+8 n^{3}\right)\left(3+10 n+12 n^{2}+8 n^{3}\right)},
$$

where $h(n):=-3+10 n-12 n^{2}+8 n^{3}=(-2+n)\left(18+4 n+8 n^{2}\right)+33>0$ for $n \geq 2$, and $h(1)=$ $3>0$. So $h(n)>0$ for $n \in \mathbb{N}$. This completes the proof of Lemma 2 .

Proof of Theorem 1 Summing the inequalities of the form

$$
g(n)-g(n+1)<\frac{1}{n^{4}}<f(n)-f(n+1)
$$

from $n$ to $\infty$ and noting that $f(\infty)=g(\infty)=0$, we have

$$
g(n)<\sum_{k=n}^{\infty} \frac{1}{k^{4}}<f(n), \quad n \geq 2 .
$$

Then, for $n \geq 2$,

$$
\frac{3}{8}\left(\frac{-3}{2 n-1}-3+10 n-12 n^{2}+8 n^{3}\right)<\left(\sum_{k=n}^{\infty} \frac{1}{k^{4}}\right)^{-1}<\frac{3}{8}\left(-3+10 n-12 n^{2}+8 n^{3}\right) .
$$

Note that

$$
\frac{3}{8}\left(-3+10 n-12 n^{2}+8 n^{3}\right)=3 n^{3}-5 n^{2}+4 n-1+\left(\frac{n(2 n-1)}{4}-\frac{1}{8}\right)
$$

and

$$
\frac{3}{8}\left(\frac{-3}{2 n-1}-3+10 n-12 n^{2}+8 n^{3}\right)=3 n^{3}-5 n^{2}+4 n-1+\left(\frac{n(2 n-1)}{4}-\frac{9}{8(2 n-1)}-\frac{1}{8}\right) .
$$


For $n \geq 5$, we have $\frac{9}{8(2 n-1)} \leq \frac{1}{8}$. Then

$$
\begin{aligned}
\left\lfloor\frac{n(2 n-1)}{4}-\frac{1}{8}\right\rfloor & \geq\left\lfloor\frac{n(2 n-1)}{4}-\frac{9}{8(2 n-1)}-\frac{1}{8}\right\rfloor \geq\left\lfloor\frac{n(2 n-1)}{4}-\frac{1}{4}\right\rfloor \\
& =\left\lfloor\frac{(2 n+1)(n-1)}{4}\right\rfloor
\end{aligned}
$$

and, for $n \in \mathbb{N}$,

$$
\left\lfloor\frac{n(2 n-1)}{4}-\frac{1}{8}\right\rfloor=\left\lfloor\frac{(2 n+1)(n-1)}{4}\right\rfloor .
$$

It follows that, for $n \geq 5$,

$$
\left\lfloor\frac{n(2 n-1)}{4}-\frac{1}{8}\right\rfloor=\left\lfloor\frac{n(2 n-1)}{4}-\frac{9}{8(2 n-1)}-\frac{1}{8}\right\rfloor=\left\lfloor\frac{(2 n+1)(n-1)}{4}\right\rfloor .
$$

Finally, we note that the above identities hold for $n=2,3,4$. Combining (2.10) and (2.11), we prove Theorem 1 .

\section{Proof of Theorem 2}

Similarly to Section 2, by the multiple-correction method we can solve the finite continued fraction approximation solution $F(n), G(n)$ of the differential equation

$$
y(n)-y(n+1)=\frac{1}{n^{5}}
$$

So we have the following inequalities.

\section{Lemma 3 Let}

$$
F(n)=\frac{9}{-2-48 n+84 n^{2}-72 n^{3}+36 n^{4}} .
$$

Then, for $n \geq 2$,

$$
F(n)-F(n+1)>\frac{1}{n^{5}}
$$

Proof

$$
\begin{aligned}
& F(n)-F(n+1)-\frac{1}{n^{5}} \\
& \quad=\frac{-1+660 n^{2}}{n^{5}\left(-1-24 n+42 n^{2}-36 n^{3}+18 n^{4}\right)\left(-1+24 n+42 n^{2}+36 n^{3}+18 n^{4}\right)} .
\end{aligned}
$$

Note that

$$
-1-24 n+42 n^{2}-36 n^{3}+18 n^{4}=(n-2)\left(60+42 n+18 n^{3}\right)+119 .
$$

Then, for $n \geq 2$, we have

$$
F(n)-F(n+1)-\frac{1}{n^{5}}>0 .
$$




\section{Lemma 4 Let}

$$
G(n)=\frac{9}{-1 / 4-48 n+84 n^{2}-72 n^{3}+36 n^{4}} .
$$

Then, for $n \geq 5$,

$$
G(n)-G(n+1)<\frac{1}{n^{5}}
$$

Proof Similarly to the proof of Lemma 3, we have

$$
\begin{aligned}
& G(n)-G(n+1)-\frac{1}{n^{5}} \\
& \quad=-\frac{1-37,536 n^{2}+2,016 n^{4}}{n^{5}\left(-1-192 n+336 n^{2}-288 n^{3}+144 n^{4}\right)\left(-1+192 n+336 n^{2}+288 n^{3}+144 n^{4}\right)} .
\end{aligned}
$$

Note that

$$
1-37,536 n^{2}+2,016 n^{4}=(-5+n)\left(64,320+12,864 n+10,080 n^{2}+2,016 n^{3}\right)+321,601
$$

and

$$
-1-192 n+336 n^{2}-288 n^{3}+144 n^{4}=(-4+n)\left(5,760+1,488 n+288 n^{2}+144 n^{3}\right)+23,039 .
$$

Then, for $n \geq 5$, the inequality $G(n)-G(n+1)-\frac{1}{n^{5}}<0$ holds.

Proof of Theorem 2 We assume that $n \geq 5$ in the following proof. Summing the inequalities of the form

$$
G(n)-G(n+1)<\frac{1}{n^{4}}<F(n)-F(n+1)
$$

from $n$ to $\infty$ and noting that $F(\infty)=G(\infty)=0$, we have

$$
\begin{aligned}
-5 & +9 n^{2}-8 n^{3}+4 n^{4}+\left(-\frac{2}{9}-\frac{n}{3}+\frac{n^{2}}{3}\right) \\
< & \left(\sum_{k=n}^{\infty} \frac{1}{k^{5}}\right)^{-1} \\
< & -5 n+9 n^{2}-8 n^{3}+4 n^{4}+\left(-\frac{1}{36}-\frac{n}{3}+\frac{n^{2}}{3}\right) .
\end{aligned}
$$

Next, for $n \geq 3$, we will prove the following identities:

$$
\left\lfloor-\frac{2}{9}-\frac{n}{3}+\frac{n^{2}}{3}\right\rfloor=\left\lfloor-\frac{1}{36}-\frac{n}{3}+\frac{n^{2}}{3}\right\rfloor=\left\lfloor\frac{(n+1)(n-2)}{3}\right\rfloor .
$$

Since

$$
\frac{(n+1)(n-2)}{3}<-\frac{2}{9}-\frac{n}{3}+\frac{n^{2}}{3}<-\frac{1}{36}-\frac{n}{3}+\frac{n^{2}}{3},
$$


it suffices to prove that

$$
\left\lfloor-\frac{1}{36}-\frac{n}{3}+\frac{n^{2}}{3}\right\rfloor=\left\lfloor\frac{(n+1)(n-2)}{3}\right\rfloor .
$$

We will consider three cases.

Case 1. If $n=3 m, m \in \mathbb{N}$, then we have

$$
\left\lfloor-\frac{1}{36}-\frac{n}{3}+\frac{n^{2}}{3}\right\rfloor=\left\lfloor\frac{(n+1)(n-2)}{3}\right\rfloor=-1-m+3 m^{2} .
$$

Case 2. If $n=3 m+1, m \in \mathbb{N}$, then we have

$$
\left\lfloor-\frac{1}{36}-\frac{n}{3}+\frac{n^{2}}{3}\right\rfloor=\left\lfloor\frac{(n+1)(n-2)}{3}\right\rfloor=-1+m+3 m^{2} .
$$

Case 3. If $n=3 m+2, m \in \mathbb{N}$, then we have

$$
\left\lfloor-\frac{1}{36}-\frac{n}{3}+\frac{n^{2}}{3}\right\rfloor=\left\lfloor\frac{(n+1)(n-2)}{3}\right\rfloor=3 m+3 m^{2} .
$$

This proves that (3.10) holds. Finally, combining (3.8) and (3.9), we prove Theorem 2.

\section{Competing interests}

The author declares that she has no competing interests.

\section{Author's contributions}

The author read and approved the final manuscript.

Received: 14 March 2016 Accepted: 11 April 2016 Published online: 04 May 2016

\section{References}

1. Lin, X: Some identities related to Riemann zeta-function. J. Inequal. Appl. 2016, 32 (2016)

2. Ohtsuka, H, Nakamura, S: On the sum of reciprocal Fibonacci numbers. Fibonacci Q. 46/47, 153-159 (2008/2009)

3. Xu, Z, Wang, T: The infinite sum of the cubes of reciprocal Fibonacci numbers. Adv. Differ. Equ. 2013, $184(2013)$

4. Zhang, W, Wang, T: The infinite sum of reciprocal Pell numbers. Appl. Math. Comput. 218, 6164-6167 (2012)

5. Zhang, H, Wu, Z: On the reciprocal sums of the generalized Fibonacci sequences. Adv. Differ. Equ. 2013,377 (2013)

6. Kilic, E, Arikan, T: More on the infinite sum of reciprocal usual Fibonacci, Pell and higher order recurrences. Appl. Math. Comput. 219, 7783-7788 (2013)

7. Komatsu, T, Laohakosol, V: On the sum of reciprocals of numbers satisfying a recurrence relation of orders. J. Integer Seq. 13, Article ID 10.5.8 (2010)

8. Komatsu, T: On the nearest integer of the sum of reciprocal Fibonacci numbers. In: Proceedings of the Fourteenth International Conference on Fibonacci Numbers and Their Applications. Aportaciones Matematicas Investigacion, vol. 20, pp. 171-184 (2011)

9. Mansour, T, Shattuck, M: Restricted partitions and q-Pell numbers. Cent. Eur. J. Math 9, 346-355 (2011)

10. Wu, Z, Zhang, H: On the reciprocal sums of higher-order sequences. Adv. Differ. Equ. 2013, 189 (2013)

11. Wu, Z, Zhang, W: The sums of the reciprocals of Fibonacci polynomials and Lucas polynomials. J. Inequal. Appl. 2012, 134 (2012)

12. Wu, Z, Zhang, W: Several identities involving the Fibonacci polynomials and Lucas polynomials. J. Inequal. Appl. 2013, 205 (2013)

13. Anantakitpaisal, P, Kuhapatanakul, K: Reciprocal sums of the tribonacci numbers. J. Integer Seq. 19, Article ID 16.2.1 (2016)

14. Cao, $X, X u, H, Y o u, X$ : Multiple-correction and faster approximation. J. Number Theory 149, $327-350$ (2015)

15. Cao, X, You, X: Multiple-correction and continued fraction approximation (II). Appl. Math. Comput. 261, 192-205 (2015)

16. Cao, X, Mortici, C: Multiple-correction and summation of the rational series. Available online at arXiv:1511.00198v1 